Military Technical College

Kobry El-Kobbah,

Cairo, Egypt

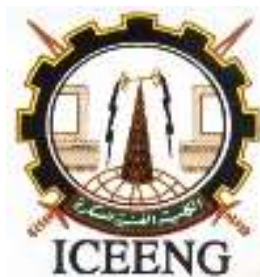

\section{Optical Design, Tolerance Analysis, and Baffling of Very} High-Resolution Satellite Telescopes 11th International Conference on

Electrical Engineering

ICEENG 2018

Mohamed Metwally*, Taher M. Bazan*, Fawzy Eltohamy*, Mahmoud Fathy*

\title{
ABSTRACT
}

The telescope design of a very high-resolution earth observation satellite is a challenging task due to several constraints, including the size, mass, performance, tolerance and environmental aspects. However, the demand for very high-resolution satellite imagery has increased day by day due to the numerous commercial and military applications. In this paper, an optical configuration for a ground sampling distance (GSD) of $25 \mathrm{~cm}$ is investigated according to a definite mission statement. The configuration represents on-axis Korsch scheme with the advantage of small conic mirror constants without higher-order parameters. In addition, a tolerance analysis utilizing a Monte Carlo algorithm is performed. The suggested baffling arrangement based on a series of point sources with different off-axis angles is derived. The size of the telescope is applicable to be launched into space by the current launch vehicles.

\section{KEY WORDS}

Very high-resolution telescope, Korsch scheme, Baffling, Tolerance Analysis.

\section{Introduction}

The remote sensing is an interesting science and technology that give the ability to get a lot of information about the earth. The more information taken from images, the high-resolution telescopes are required. Therefore, improving the resolution of the remote-sensing satellite images is a critical issue. However, this target is considered as a challenge for the designers $[1,2]$.

With the huge technology revolution, many ideas take place to improve the design techniques of the earth observation telescopes. For example, the Korsch configuration is chosen as the best design concept for a compact optical telescope according to a comparative studies presented by Italian Space Agency [3] and Centre National d'Etudes Spatiales CNES [4]. On the other hand, off-axis three mirror anastigmatic (TMA) configuration provides a proper solution to satisfy the requirements of the high-resolution and wide swath [5]. If compared to Korsch schemes, TMA faces more challenges in design, measurements and alignment

* Egyptian Armed Forces

${ }^{* *}$ Benha University 
accuracy, according to the studies of Beijing Institute of Space Mechanics and Electricity (BISME) [5]. As a result, the Korsch scheme will be adopted in this paper as base line for the design of very high-resolution satellite telescope.

The mission statement in this paper is to utilize a telescope, with a panchromatic ground sampling distance (GSD) of $0.25 \mathrm{~m}$ and a swath width of $8 \mathrm{Km}$, within a satellite orbiting around the Earth in a sun-synchronous orbit with an inclination of $98.5^{\circ}$ at $500 \mathrm{~km}$ altitude. The modulation transfer function (MTF) of the telescope should be not less than 0.25 including the optical manufacturing tolerance issue. The paper is organized as follows. The key design parameters of the telescope are investigated in Section 2. The design of the proposed optical scheme based on Korsch configuration is presented in Section 3. The tolerance analysis of the proposed system is investigated in Section 4 whereas the associated baffling design is derived in Section 5. Finally, Section 6 summarizes the conclusion.

\section{Main Design Parameters}

Starting from the mission statement, the main telescope parameters can be easily investigated as follows; the focal length $f$ of the telescope in terms of satellite altitude $H$, detector pixel size $\Delta$, and the GSD is given by [6]

$$
f=\frac{H \cdot \Delta}{G S D}
$$

Using a detector with pixel size of $10 \mu \mathrm{m}$ [7] resulting in a focal length of $20 \mathrm{~m}$ and this large focal length is expected since we are dealing with the very high-resolution task. According to the diffraction theory, the clear aperture $D$ of the telescope is defined based on Rayleigh criterion as follow [8]

$$
D=\frac{1.22 \lambda f}{\Delta}
$$

where $\lambda$ is the central wavelength of the panchromatic sensor. If the maximum wavelength is $0.4 \mu \mathrm{m}$ and the minimum wavelength is $0.9 \mu \mathrm{m}$, then, the required clear aperture diameter will be $1400 \mathrm{~mm}$ and the telescope F-number is $\sim 14.3$. Although the value of $D$ is quite high, many launchers have a wide fairing to accommodate such a telescope. For example, the Russian rocket Soyuz-2b with a payload fairing diameter of $3 \mathrm{~m}$ may be utilized as a launcher. To achieve the required swath width, the field of view of the telescope should be calculated as follows

$$
F o V=2 \tan ^{-1} \frac{S w a t h}{2 H}
$$

Thus, the FoV of $0.9167^{\circ}$ is required to ensure a swath width of $8 \mathrm{Km}$ at $\mathrm{H}=550 \mathrm{Km}$. However, the design of the telescope should comply with these main parameters.

\section{Korsch Telescope Design}

\section{A. Optical scheme}

The proposed Korsch configuration is illustrated in Fig 1. The Korsch scheme consists of three on-axis aspheric mirrors (primary mirror $\mathrm{M} 1$, secondary mirror $\mathrm{M} 2$, and third mirror M3) with small conic constants. Two flat mirrors (M4 and M5) are employed as folding mirrors. The main characteristics of the utilized mirrors are 
summarized in Table 1. Note that the conic constants of $M 1, M 2$, and $M 3$ are applicable from the manufacturing point of view and the distance between M2 and M3 is $~ 3430 \mathrm{~mm}$. In addition, the alignment of the proposed configuration is applicable because the three mirrors are on the same axis.

Table 1: Korsch Optical Design Parameters (units in mm)

\begin{tabular}{|c|l|l|l|l|l|}
\hline Element & \multicolumn{1}{|c|}{ Radius } & Thickness & \multicolumn{1}{|c|}{ Diameter } & \multicolumn{1}{c|}{ Conic } & \multicolumn{1}{|c|}{ Y-shift } \\
\hline M1 & 4914.905 & 1961.331 & 1400 & -0.97262 & 0 \\
\hline M2 & -1301.961 & 3429.298 & 331 & -2.36151 & 0 \\
\hline M3 & 1772.163 & 1069.599 & $270 \times 120$ & -0.53112 & -120 \\
\hline M4 & Infinity & 771.7647 & 145 & 0 & 0 \\
\hline M5 & Infinity & 888.274 & $160 \times 50$ & 0 & 90 \\
\hline
\end{tabular}

\section{B. Performance assessment}

The next discussion is devoted to assessing the performance of the proposed Korsch telescope in terms of the major metrics such as MTF, spot diagram, field curvature and distortion. The performance of the Korsch scheme in terms of MTF is shown in Fig 2. It is obvious that MTF reaches 0.4 at a Nyquist frequency of $50 \mathrm{Lp} / \mathrm{mm}$ for the entire FoV even for sagittal or tangential directions.

Fig 3 shows the spot diagram of the proposed telescope for different angles within the required field of view. It is clear that the images at focal plane are confined to the pixel size of $10 \mu \mathrm{m}$. The associated field curvature and distortion are illustrated in Fig. 4. It is clear that the maximum field curvature is $0.047 \mathrm{~mm}$ and the maximum distortion is $0.368 \%$.

\section{Tolerance Analysis}

Generally, tolerance describes the visibility from the manufacturing point of view. The tolerance aspect can be described by the tolerance factor [4]. Taking the same reference tolerance budget presented in [4], the tolerance factor can be defined as the ratio between the resulted tolerance budget of the proposed design to the reference one. The more tolerance factor, the more manufacturing stability, i.e., the system can withstand more manufacture tolerance. Let $T_{X}$ and $T_{Y}$ are the tilting tolerance budget around $X$ and $Y$-axes, respectively. Similarly, let $D_{x}$, Dy and $D_{z}$ are the tolerance budget for displacement in the directions $\mathrm{X}, \mathrm{Y}$, and Z-axes, respectively. Consider $\Delta \mathrm{R}$ is the tolerance in radius of curvature. Table 2 shows the tolerance budget for the proposed Korsch scheme based on the Monte Carlo algorithm with 1000 trials.

As a comparison, the proposed Korsch configuration gives a tolerance factor of 0.347 which is better than 0.27, the best tolerance factor achieved in reference [4]. Note that $90 \%$ of the trials achieve MTF greater than 0.348 . Thus, the MTF including the tolerance issue satisfy the required MTF in the mission statement.

\section{Korsch Baffling System}


The baffling design is an important task to remove or minimize the stray light that may reach the photodetector. To achieve such a goal, the stray light power should be less than the detector noise power (dark current power) to get the best performance of the

Table 2: The Tolerance Budget

\begin{tabular}{|c|c|c|}
\hline Objective Components & Tolerances type & Tolerances budget \\
\hline \multirow{3}{*}{$\begin{array}{c}\text { Mirror 1 } \\
\text { (Reference) }\end{array}$} & $T_{X} \& T_{Y}$ & $1 "$ \\
\hline & $D_{x} \& D_{Y}$ & 20 \\
\hline & $\Delta R$ & $0.8 \mu \mathrm{m}$ \\
\hline \multirow{4}{*}{ Mirror 2} & $T_{X} \& T_{Y}$ & $5 "$ \\
\hline & Dx \& Dy & $20 \mu \mathrm{m}$ \\
\hline & $\mathrm{Dz}$ & $0.4 \mu \mathrm{m}$ \\
\hline & $\Delta \mathrm{R}$ & $1.4 \mu \mathrm{m}$ \\
\hline \multirow{4}{*}{ Mirror 3} & $T_{X} \& T_{Y}$ & $4 "$ \\
\hline & $D_{x} \& D_{Y}$ & $15 \mu \mathrm{m}$ \\
\hline & $\mathrm{Dz}$ & $7.5 \mu \mathrm{m}$ \\
\hline & $\Delta \mathrm{R}$ & $6.5 \mu \mathrm{m}$ \\
\hline \multirow[b]{2}{*}{ Mirror 4} & $\mathrm{Dz}$ & $87 \mu \mathrm{m}$ \\
\hline & $\Delta \mathrm{R}$ & $\begin{array}{c}\mathrm{R}=3 \times 10^{7} \mathrm{~mm} \pm \\
0.015 \mu \mathrm{m}\end{array}$ \\
\hline \multirow[b]{2}{*}{ Mirror 5} & $\mathrm{Dz}$ & $45 \mu \mathrm{m}$ \\
\hline & $\Delta R$ & $\begin{array}{c}\mathrm{R}=7 \times 10^{6} \mathrm{~mm} \pm \\
0.714 \mu \mathrm{m}\end{array}$ \\
\hline \multirow{2}{*}{ Focal block } & Dx \& Dy & $45 \mu \mathrm{m}$ \\
\hline & $\mathrm{Dz}$ & $30 \mu \mathrm{m}$ \\
\hline Tolerance factor & & 0.347 \\
\hline
\end{tabular}

optical system [9]. The received stray light power on the detector is usually described by the point source transmittance (PST) [10]. PST is defined as the irradiance at the reference plane (photodetector plane in our case) at a particular angle divided by the input irradiance for the same angle. The baffling design follows the same procedures presented in [10]. The starting point is to determine the critical elements; M1 opening hole, M3, M4, and M5. To minimize the number of these critical elements, blacked baffling plates (stops) will be used to reject the stray light as shown in Fig 5.

As shown in Fig 5, plate 1 blocks the stray light that may be reflected from M3 and reaches the focal plane whereas plate 2 blocks the stray light coming from M1 opening hole or M4. Therefore, the remaining critical element is M5. The next step is devoted to determine the illuminated objects. It is obvious that M1 opening hole is the key source of light of the Korsch system to enter the back box. To minimize the illuminated object seen by M5, M4 backside is already acting as a baffling plate; preventing the stray light to reach M5.

The next step is to determine all the rays pass from M1opening hole to incident on M5. As shown in Fig. 6a, there are three possible critical optical paths; paths no.1, 2, 
and 3. In order to minimize the effect of these critical paths, three actions should be taken. Adding vans to M1 opening hole edges to minimize the effect of critical path no. 1 and plates $3 \& 4$ for critical paths no. 2 and 3 as illustrated in Fig $6 \mathrm{~b}$. It is worth mentioning that the baffling design here assumes more than two reflections on the black surfaces will diminish the optical stray light power. Also, adjusting M3 dimension exactly with the FoV is necessary because increasing of M3 dimension will increase the possibility of stray light effect.

Now, the design of vans (numbers, heights and positions) is derived through the following procedure. Assume $r$ be the radius of the main tube (containing M1, and $\mathrm{M} 2$ ), a be the $\mathrm{M} 1$ radius, and $S$ be the length of the main tube. According to the variables illustrated in Fig. $7, \mathrm{x}_{\mathrm{n}}$ is the positions of the $n$th van while $\mathrm{Zn}$ is the position of the reflected ray after $n$th van. Starting with $\mathrm{x}_{0}=0$, yo can be easily calculated according to the following relation

$$
y_{0}=a+S \tan ^{-1}(F o V)
$$

Then, $x_{1}$ and $y_{1}$ can be calculated by intersecting the line $L_{1}$ and FoV so that

and

$$
x_{1}=\frac{\mathrm{S}\left(r_{0}-y_{0}\right)}{2 \mathrm{a}+r_{0}-y_{0}}
$$

$$
y_{1}=\frac{a\left(r_{0}-y_{0}\right)-r_{0} y_{0}+r_{0}^{2}}{2 \mathrm{a}+r_{0}-y_{0}}
$$

From figure trigonometry, the intersection of the line $L_{1}$ and the tube is given by

$$
Z_{1}=\frac{X_{1}\left(y_{0}+r\right)}{r+y_{0}-Y_{1}}
$$

In general, the location of intersection between line $L_{n}$ and the tube is expressed in terms of $x_{n}$ and $y_{n}$ as

$$
\mathrm{Z}_{\mathrm{n}}=\frac{\mathrm{X}_{\mathrm{n}}\left(\mathrm{y}_{0}+\mathrm{r}\right)}{\mathrm{r}+\mathrm{y}_{0}-y_{\mathrm{n}}}
$$

By solving the line $K_{n+1}$ and field of view, then

and

$$
X_{n+1}=\frac{S^{2}\left(r-y_{0}\right)+S \cdot Z_{n}\left(a+y_{0}\right)}{S\left(2 \cdot a+r-y_{0}\right)-Z_{n}\left(a+y_{0}\right)}
$$

$$
Y_{n+1}=\frac{S \cdot r^{2}-a^{2} \cdot Z_{n}+\text { S.a.r }- \text { a.r. } Z_{n}+y 0\left(a \cdot Z_{n}-S \cdot a-S \cdot r+r \cdot Z_{n}\right)}{2 \cdot S \cdot a+S \cdot r-S \cdot y_{0}-a \cdot Z_{n}+y_{0} \cdot Z_{n}}
$$

By repeating the same sequence, the positions and heights of vans in the main tube are presented in Table 3 where the distance is measured from the primary mirror. Fig 
Table 3: Vans heights and positions within the main tube (the unit in $\mathrm{mm}$ )

\begin{tabular}{|l|c|c|c|c|c|c|c|c|c|c|}
\hline & 1 & 2 & 3 & 4 & 5 & 6 & 7 & 8 & 9 & 10 \\
\hline Distance & 51 & 181 & 308 & 433 & 556 & 676 & 793 & 908 & 1021 & 1131 \\
\hline Y-Radius & 750 & 753 & 755 & 757 & 759 & 761 & 763 & 765 & 767 & 769 \\
\hline & 11 & 12 & 13 & 14 & 15 & 16 & 17 & 18 & 19 & 20 \\
\hline Distance & 1239 & 1345 & 1448 & 1550 & 1649 & 1746 & 1840 & 1933 & 2024 & 2113 \\
\hline Y-Radius & 771 & 773 & 775 & 777 & 778 & 780 & 782 & 783 & 785 & 786 \\
\hline
\end{tabular}

8 shows the position of the two groups of sharp edge vans with $90^{\circ}$ tilting where the first group at the main tube and the second at the M1 opening hole.

Fig 8 shows the position of the two groups of sharp edge vans with $90^{\circ}$ tilting where the first group at the main tube and the second at the M1 opening hole. Finally, the stray light power calculation for a series of off-axis cases of the point sources will be performed to check the overall baffling performance. Baffling simulation and evaluation are performed by Zemax [12].

\section{Simulation Assumptions:}

- The incident light on the telescope aperture is simulated as parallel rays with different inclination angles up to $80^{\circ}$.

- The telescope structure is coated with Chemglaze Z306 with silica powder as a black material since it gives a less than $7 \%$ Lambertian reflection in all directions [11].

- All mirrors are coated by Al with a reflection coefficient of $94 \%$ [13].

However, based on the Landsat document [14], the typical value of the radiance reflected from the Earth is $40 \mathrm{~W} / \mathrm{m} 2 \mu \mathrm{m}$ sr within the spectral band of 4500 to 5150 $\mathrm{nm}$ and $23 \mathrm{~W} / \mathrm{m} 2 \mu \mathrm{m} . \mathrm{sr}$ for the spectral band of 5000 to $6800 \mathrm{~nm}$, and these two bands cover the proposed telescope bands. Therefore, the irradiance incidents on the Korsch entrance pupil can be expressed according to the following relation

$$
E_{a p}=\frac{L_{E} \times \Delta \lambda \times A_{a p}}{H}
$$

where $L_{E}$ is the Earth irradiance and $A_{a p}$ is aperture area. Thus, $E_{\mathrm{ap}}=2.075 \times 10^{-5}$ $\mathrm{W} / \mathrm{m}^{2}$. Also, the satellite bus which will carry the proposed telescope must satisfy the requirement that no shiny objects in the angle $\pm 50^{\circ}$ exist around the optical axis of the telescope. Additional external sun baffle is recommended to be used and fixed on the satellite panel. Nevertheless, the design of sun baffle is out of the scope of this study.

The simulation output covering all the incident angles is the distribution of PST as presented in Fig 9. According to the photodetector data sheet [7], it is clear that the design of the baffling system is affirmed since the stray light power is less than the detector sensitivity.

Finally, to show the advantages of the proposed telescope, a comparison between the design of the proposed telescope and that presented by $\mathrm{CXCI}$ program (related to 
the French Space Agency) planned to be launched in 2020-2022 [4] is given in Table 4. It is clear that the proposed telescope is superior in most issues. However, to achieve more compactness to the proposed telescope, folding mirrors can be used without scarifying the performance but at the expense of the number of optical elements.

Table 4: A comparison between the proposed and $\mathrm{CXCl}$ optical designs

\begin{tabular}{|c|c|c|}
\hline $\begin{array}{ll}\text { Parameter } & \text { Type } \\
\end{array}$ & Proposed telescope & CXCI design [4] \\
\hline Resolution & $0.25 \mathrm{~m} @ 500 \mathrm{Km}$ & $0.3 \mathrm{~m} @ 700 \mathrm{Km}$ \\
\hline F-number & $F / 14.3$ & $F / 20$ \\
\hline MTF & 0.348 & 0.34 \\
\hline Tolerance factor & 0.347 & 0.27 \\
\hline X distortion & $0.368 \%$ & $0.9 \%$ \\
\hline Y distortion & $0.16 \%$ & $0.3 \%$ \\
\hline Number of optical elements & 5 & 7 \\
\hline M1-M2 distance & $1.961 \mathrm{~m}$ & $1.8 \mathrm{~m}$ \\
\hline Total axial telescope length & $3.43 \mathrm{~m}$ & $2.6 \mathrm{~m}$ \\
\hline
\end{tabular}

\section{Conclusion}

The optical design of a very high-resolution telescope with a GSD of $0.25 \mathrm{~m}$ suitable for the next-generation earth observation system has been investigated. Several subjects are explored in details including the optical performance and tolerance analysis. Furthermore, the associated baffling system is derived and accurately simulated. The optical performance shows that the MTF of the proposed Korsch configuration is 0.35 with F-number of 14.3, and low field curvature and distortion values. The proposed telescope shows a powerful design in many aspects if compared to the recent studies.

\section{Figures}

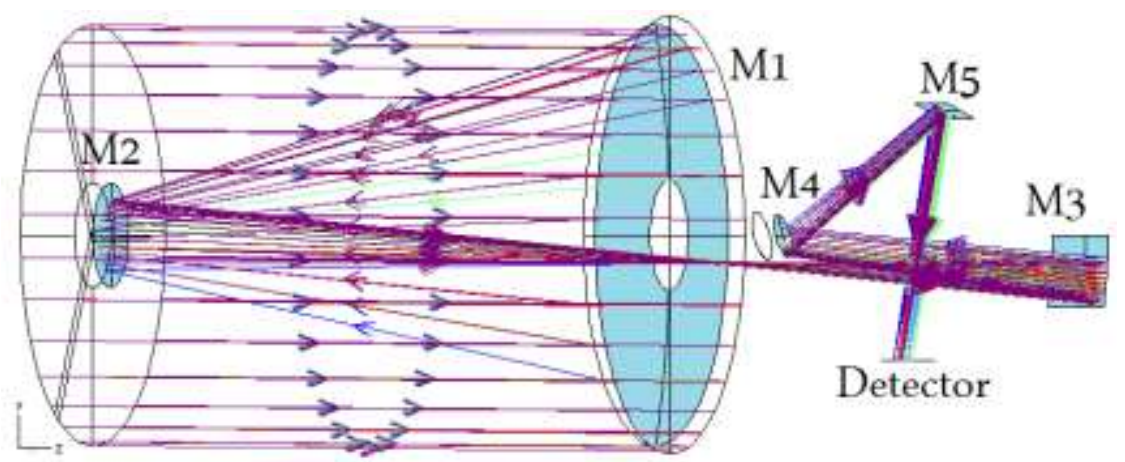

Fig 1 Layout of the proposed Korsch telescope 


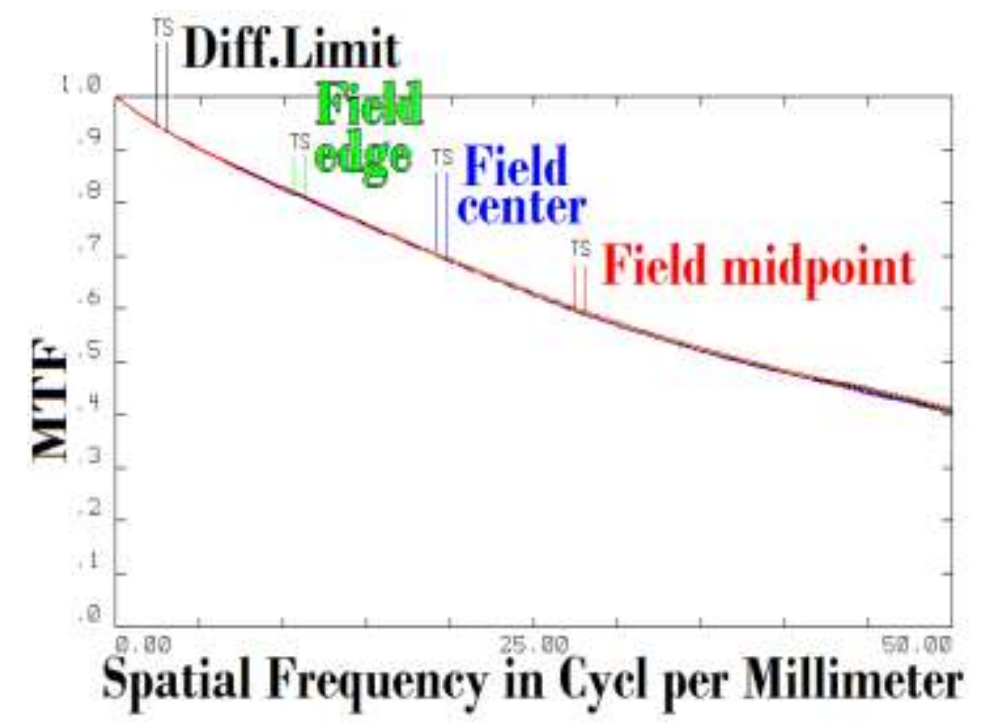

Fig 2 MTF curve for the proposed Korsch telescope

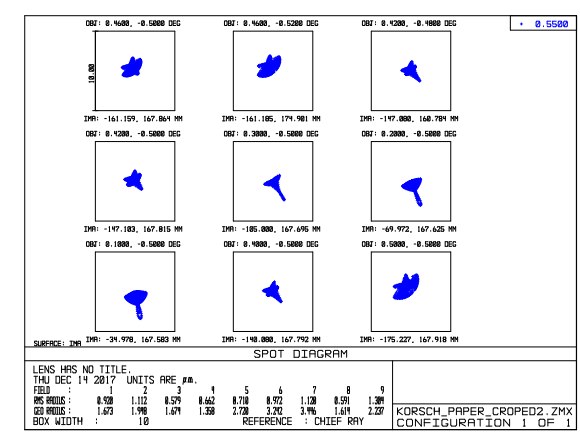

Fig 3 The spot diagram of the Korsch system 


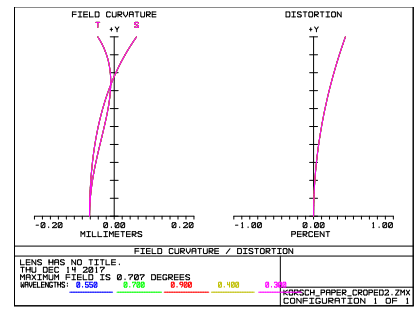

Fig 4 Field curvature and distortion

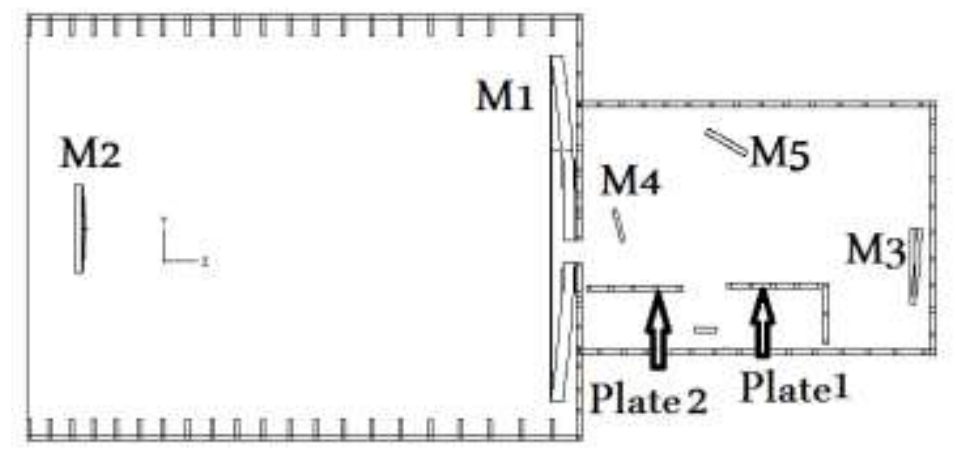

Fig 5 The position of plate 1 and 2

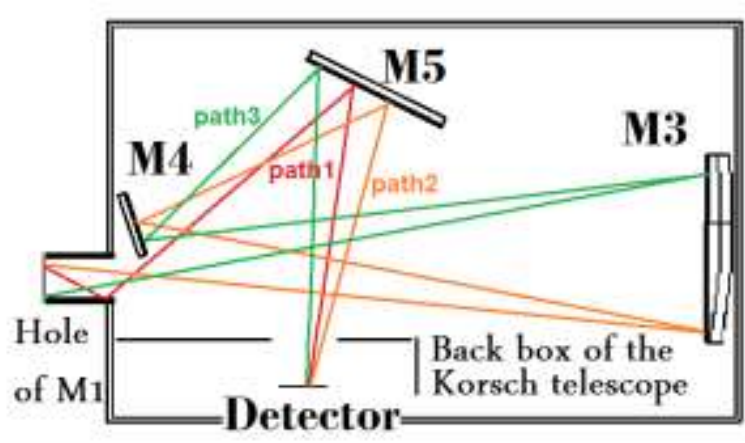

(a)

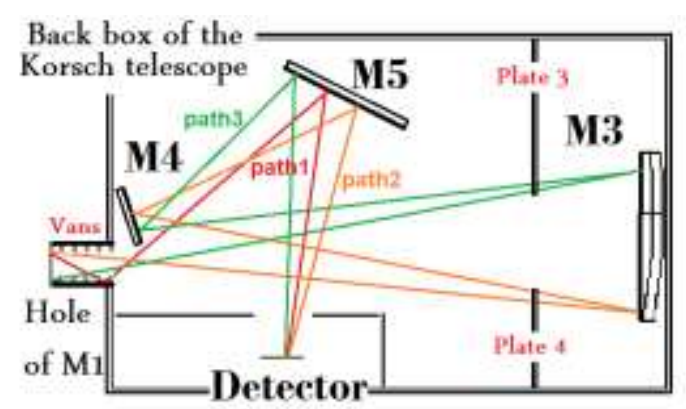

(b)

Fig 6 The baffling design (a) Critical paths of the system (b) Critical paths removal through plates 


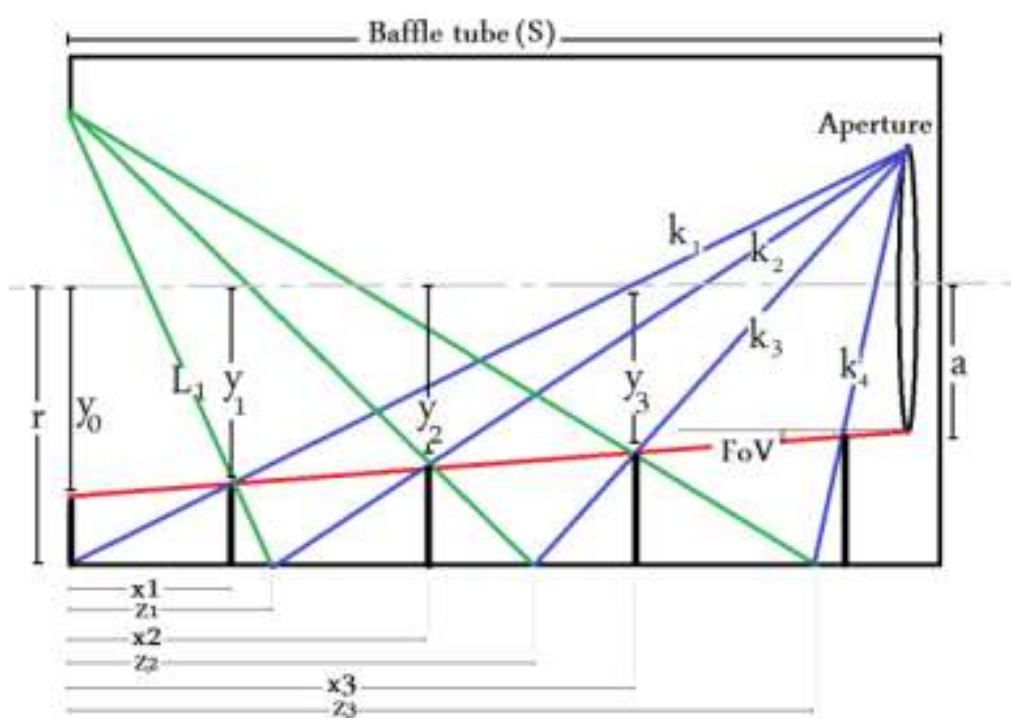

Fig 7 The vans parameters

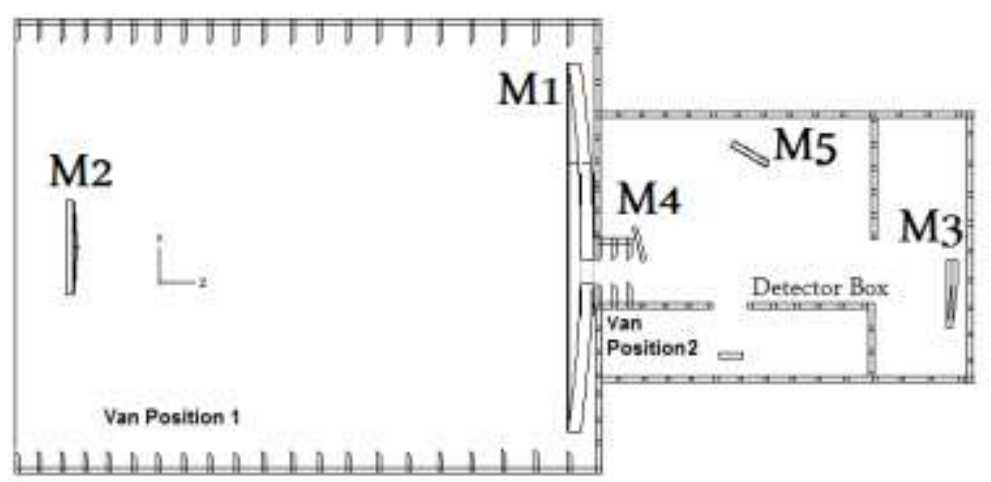

Fig 8 Description of final baffling layout

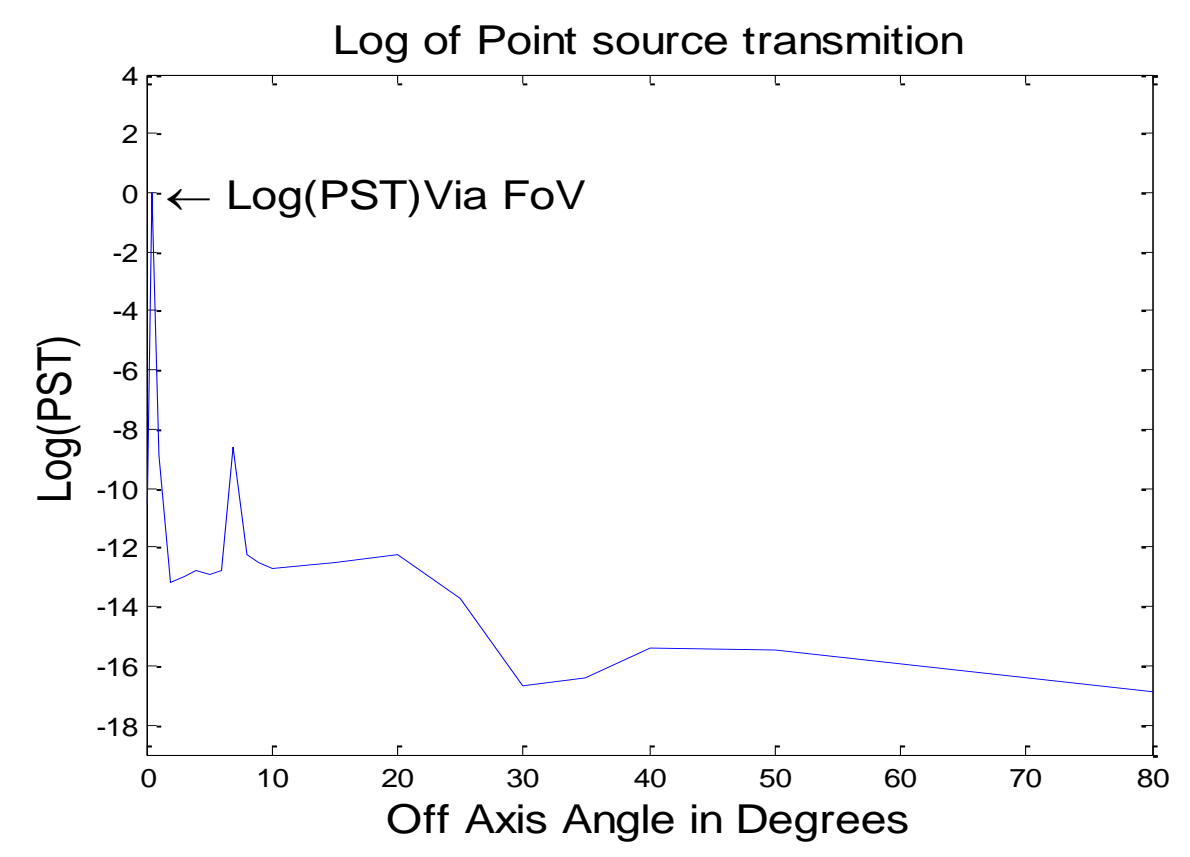

Fig 9 The simulation output of the Korsch baffling system 


\section{References}

[1] R. Woodruff, T. Hull, S. Heap, W. Danchi, S. Kendrick, and L. Purves, "Optical design for CETUS: a wide-field $1.5 \mathrm{~m}$ aperture UV payload being studied for a NASA probe class mission study, "Proc. SPIE, Astronomical Optics: Design, Manufacture, and Test of Space and Ground Systems, 104011P, 2017.

[2] J.-M. Delvit, C. Thiebaut, C. Latry, G. Blanchet, "Wave front sensing for next generation earth observation telescope," International Conference on Space Optics (ICSO), France, 2016.

[3] F. Simonetti, A. Romoli, P. Mazzinghi, and V. Brantina, "Reflecting telescopes for an orbiting high-resolution camera for Earth observation," Opt. Eng. vol. 45, pp. 053001, 2006.

[4] V. Costes, G. Cassar, L. Escarrat, and S. Conseil "Optical design of a compact telescope for the next generation earth observation system" Proceedings of International Conference on Space Optical Systems and Application (ICSOS), 2012.

[5] B. FAN, W.-J. Cai, Y. Huang, "Design and test of a high performance off-axis TMA telescope" Proceedings of International Conference on Space Optical Systems and Application (ICSOS), 2012.

[6] N. Wanna, "Design of reflective optical systems," M.Sc. thesis, Faculty of North Carolina State University, pp. 26-27, 2006.

[7] ON Semiconductor "KLI-4104 Data sheet", www.onsemi.com, 2015 - Rev. 5.

[8] N. Menn, "Practical optics" Elsevier Inc, pp. 60-64, 2004.

[9] L. Scaduto, É. Carvalho, L. Santos, F. Yasuoka, M. Stefani, and J. Castro, "Baffle design and analysis of stray-light in multispectral camera of a Brazilian satellite", Annals of Optics, XXIX ENFMC, 2006.

[10] Robert P. Breault, "Control of Stray Light", in "Handbook of Optics", vol. 1, McGraw-Hill, New York, 1995, Chapter 38.

[11] R. Breault and S. Pompea, "Black surfaces for optical systems," in "Handbook of Optics", vol. 2, Second Edition, McGraw-Hill, New York, Chapter 37, 1995.

[12] A. Buffington, B. Jackson, and P. Hick "Calculations for, and laboratory measurements of a multistage labyrinthine baffle for SMEI," Proceedings of SPIE, vol. 4853, 2003.

[13] A. foster, T. Armstrong, P. Chadwick, and M. Held, "Dielectric coatings for IACT mirrors," International Cosmic Ray Conference, Rio De Janeiro, 2013.

[14] NASA internet site "Landsat 8 reference parameters" internet PDF document

[15] https://sustainablelandimaging.gsfc.nasa.gov/rfi/Landsat_8_Reference_Paramete rs.pdf. 